\title{
Elasticity and glassy dynamics of dense emulsions
}

\author{
Frédéric Cardinaux $^{*, \dagger}$, Thomas G. Mason ${ }^{\dagger, * *}$ and Frank Scheffold* \\ * Department of Physics, University of Fribourg, CH-1700 Fribourg, Switzerland. \\ $\dagger$ Department of Chemistry and Biochemistry, University of California, Los Angeles, CA 90095, USA. \\ ${ }^{* *}$ Department of Physics and Astronomy, University of California Los Angeles, Los Angeles, CA 90095, USA
}

\begin{abstract}
We present a rheology and light scattering study of the dynamical properties of dense emulsions at volume fractions ranging from viscous liquid to deeply jammed states. From temporally and spatially revolved dynamic light scattering we obtain detailed information about the internal dynamics. Our measurements thus allow a direct study of heterogeneous nature of the relaxation processes involved in jammed assembly of emulsion droplets.
\end{abstract}

Keywords: colloids,emulsions, jamming, glassy dynamics, light scattering, rheology

PACS: 82.70.-y, 42.30.Ms, 64.70.Pf, 05.40.-a

\section{INTRODUCTION}

An emulsion is a dispersion of a liquid in another, immiscible liquid. Modern emulsification techniques allow to produce monodisperse emulsions with sizes ranging from several microns down to few tenth of nanometers $[1,2]$. Coalescence can be prevented by coating the droplet surfaces with additives, thus rendering the system long-lived. Emulsions are ideal model systems to study glassy dynamics and jamming since they offer a wide range of accessible and controllable parameters: Emulsion droplets are well-defined building blocks with sharp boundaries. Monodisperse droplets can be obtained in sufficient quantities by Bibette's method of fractionation [3, 4]. In stable emulsions neighbouring particles do not interpenetrate, in contrast to soft polymer spheres [5,6]. The interaction potential is known and can be tuned from soft to hard, repulsive to attractive and if required emulsions can be optically index matched or contrast enhanced, density matched and fluorescently labeled $[3,7,8,9,10,11,12]$.

As the volume fraction is increased well above $\phi=0.5$ emulsions form solid phases. If crystallisation is suppressed due to a finite polydispersity $\sigma_{D} / \bar{D} \geq 0.10$ it is generally assumed that the system enters a disordered glassy phase at a volume fraction of about $\phi_{G} \simeq 0.58$ [7, 8] (see Figure 1). In this regime, since the droplets are not yet deformed, the behavior of repulsive emulsion droplets is very similar to the one of hard spheres. This is generally valid to a very good approximation since droplet deformation typically requires forces orders of magnitude higher than the entropic forces, due to crowding, acting in this regime. However, the elasticity rapidly increases once the free volume drops to zero and the droplets exert direct contact forces onto each other. Now, in contrast to hard spheres, the elastic modulus of emul- sions does not diverge, and disordered emulsion droplets can be prepared at volume fractions as high as $\phi=0.8$ and above. Indeed, due to the liquid nature of both the continuous and the dispersed phase, droplets can deform when packed together while keeping the droplet volume unchanged. This situation typically occurs at volume fractions beyond $\phi_{J}$, the jamming or random close packing of hard spheres [13,14], where deformation results from the contact forces exerted by individual droplets on each other. As a consequence, the physical properties of dense emulsions strongly depends on whether droplets deform or not. Most notably, it impacts on the rheology of the non-equilibrium states found at high volume fractions [7, 8]. Above random close packing the elasticity of emulsions is surprisingly well captured using a network spring model $[7,15,16]$. The model accounts for energy storage at the droplet's interface when deformed and assumes the presence of a stress bearing network having density that varies with $\phi$. Despite the dominantly elastic response, rheological studies showed that dissipative processes remain important even for highly compressed emulsions [16].

He we present a study of the dynamic properties of dense emulsions at volume fraction ranging from $0.4<$ $\phi<0.72[2,7,8]$. We use an experimental approach that allows temporally and spatially revolved dynamic light scattering in highly turbid media $[17,18,19]$.

\section{MATERIAL AND METHODS}

\section{Preparation of stable emulsion droplets}

We study silicon oil-in-water emulsions stabilized by $8 \mathrm{mM}$ sodium dodecylsulfate (SDS). Oil droplets are prepared by shear rupturing of a crude emulsion and subse- 

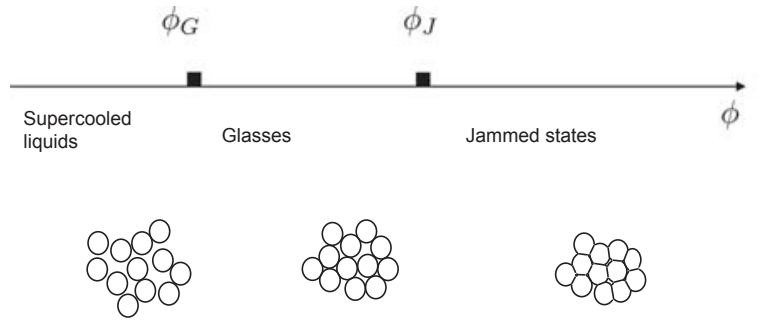

FIGURE 1. Generic scenario of the glass and jamming transition for dense suspensions of hard spheres in the case when crystallisation is suppressed [20]. A supercooled liquid enters a solid glassy phase at about $\phi_{G} \simeq 0.58$ well below random close packing at $\phi_{J} \simeq 0.64$. Deformable spheres, such as emulsion droplets, can be driven into higher density states via a shape deformation. In this regime the Laplace pressure of the droplet dictates the direct interaction forces between neighboring droplets.

quent size segregation using depletion induced sedimentation [4]. The resulting suspension is composed of oil droplets having an average diameter $\bar{D}=2.6 \mu \mathrm{m}$ and a polydispersity $\sigma_{D} / \bar{D}=0.10$. The Fig. 2 show a confocal microscopy picture of a dyed emulsions. The size distribution $P(D)$, derived from video microscopy, is displayed as well as the gaussian fit from which $\bar{D}$ and $\sigma_{D}$ are obtained. In order to minimize the effect of gravity, we match the density of the continuous phase to that of the oil using a mixture of water and heavy water. Based on centrifugation experiments, we estimate the gravitational Péclet number to $P e=m g \bar{D} / 2 k_{B} T \approx 10^{-2}$, where $m$ is the buoyant mass and $g$ the gravitational acceleration. We evaporate the continuous phase to determine the weight fraction of the oil and associate it to the volume fraction. The presence of charged surfactant on the droplet's surface leads to a screened Coulomb repulsion between droplets. We therefore consider an effective volume fraction $\phi_{\text {eff }}=\phi(1+3 h / \bar{D})$ which accounts for a minimal separation distance between interface of $h \simeq 17 \mathrm{~nm}[16]$.

\section{Rheology experiments}

The mechanical properties of the emulsions are characterized using a stress-controlled rheometer (MCR300 from Paar Physica) having a cone and plate geometry and equipped with a solvent trap. After loading the samples, we apply a pre-shear at a shear rate of $\dot{\gamma}=300 \mathrm{~s}^{-1}$ for 60 s and wait for a period of 300 s before starting measurements. We determine the regime of linear response to a shear perturbation and we measure the elastic and loss modulus, $G^{\prime}$ and $G^{\prime \prime}$, at frequencies ranging from $0.5-300 \mathrm{rad} / \mathrm{s}$.

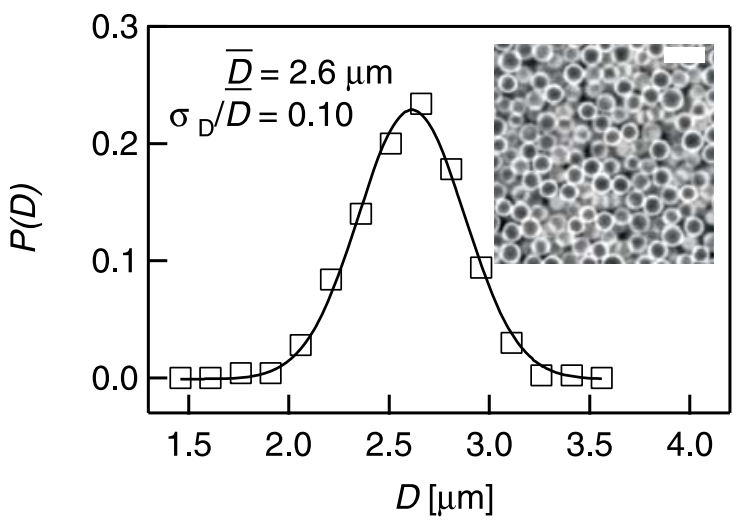

FIGURE 2. Size distribution $P(D)$ of emulsion droplets having an average diameter of $\bar{D}=2.6 \mu \mathrm{m}$ and a polydispersity $\sigma_{D} / \bar{D}=0.10$. The line is a Gaussian fit to the data. Inset: confocal image of the same droplets died using Nile Red. The scale bar is $5 \mu \mathrm{m}$.

\section{Light scattering experiments}

Most oil-in-water emulsions are very opaque owing to the strong multiple scattering of visible light. As a consequence the dynamics of the system cannot be assessed using traditional dynamic light scattering methods or video microscopy. In principle emulsions can be cleared almost entirely by refractive index matching [1]. However it is very challenging to both match both the droplets refractive index and density at the same time. We therefore decided to use diffusing wave spectroscopy (DWS) for the present study. DWS is a photon correlation technique that analyzes directly the fluctuations of multiply scattered light. We adopt a detection layout wherein the sample is illuminated with a expanded laser light source and the backscattered intensity pattern $I(\mathbf{r})$ is recorded at a close distance from the sample's surface [17]. The experimental setup is schematically illustrated in the Fig. 3. We use a 50mm-objective to do one-toone mapping of the backscattered intensity onto chargecoupled device sensor (Pixelfly from PCO, $640 \times 480$ pixels having a size of $9.9 \mu \mathrm{m}$ ). An analyzer selects a single polarization component of $I(\mathbf{r}, t)$ and the size of the detected speckles is adjusted to twice the pixel size using the objective's aperture. The sample illumination consists of speckles created by a focused laser (Coherent Verdi V5, $\lambda=532 \mathrm{~nm}$ ) impinging on a ground glass and collected by a lens in order to form a speckle-beam as described in [17, 19]. Periodic modulation of the ground glass using a linear [19] or rotary [17] electric motor can be applied to improve the signal to noise ratio for spatially resolved DWS. Finally, we characterize the fluctuations of the backscattered intensity by computing the instantaneous structure coefficient between intensity pat- 


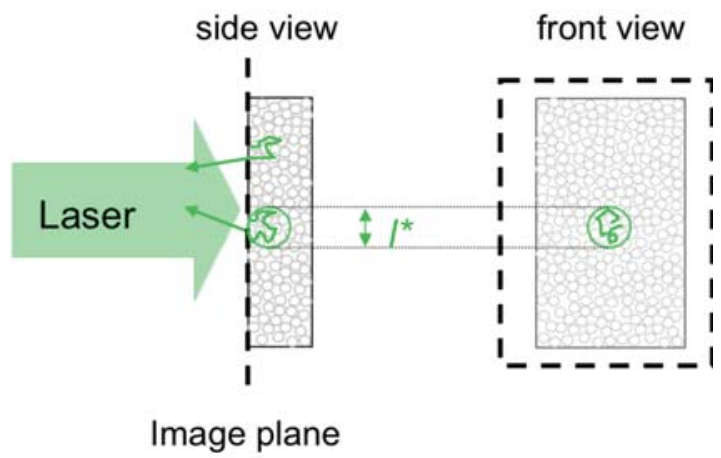

FIGURE 3. Experimental setup for imaging of diffuse laser light reflected from a turbid sample. The incident laser light can be scrambled by a ground glass (no shown) to probe statistically independent optical configurations [17]. Scattered light is recorded in the image plane with a digital camera.

tern separated by an elapsed time $\tau$ :

$$
d(\mathbf{r}, \tau, t)=|i(\mathbf{r}, t)-i(\mathbf{r}, t+\tau)|^{2},
$$

where $i(\mathbf{r}, t)=I(\mathbf{r}, t) /\langle I(t)\rangle_{\mathbf{r}}$ is the intensity pattern normalized by its spatial average value and $\tau=n \cdot t_{c}$ is an integer multiple of the $t_{c}$. For the spatially averaged experiments $t_{c}=1 / 50 \mathrm{~s}$ is set by the camera frame rate. For the spatially resolved experiments we select either $t_{c}=2.6 \mathrm{~s}$ or $t_{c}=5.2 \mathrm{~s}$ which in this case is equal to the aforementioned period of the speckle modulation.

The intensity structure function (ISF) as a direct measure of the dynamic activity has several advantages over the commonly used intensity correlation function (ICF) $g_{2}(t, \tau)=\langle[i(t) i(t+\tau)]\rangle$. While both quantities are directly related in the limit of perfect measurement statistics $d(t, \tau)=2\left[g_{2}(t, 0)-g_{2}(t, \tau)\right]$, the ISF is known to outperform the ICF in accuracy when the collection time is limited and further the ISF is less sensitive to low frequency noise or drifts [21].

Our experimental layout allows us to study the structure coefficient both spatially averaged as a well as spatially resolved. The resolution is in this case however limited by the multiple scattering process as indicated in Figure 3. Briefly, the propagation of light in the multiple scattering regime can be described as a random walk of photons with an effective step length of $l^{*}$, also known as the transport mean free path. For sufficiently large number of steps light propagation is diffusive. Diffusely reflected light penetrates the sample up to a depth of (a few) $l^{*}$ and when leaving the sample spreads out over an area of roughly $10\left(l^{*}\right)^{2}[17,22,23]$. In turn, light detected at a given point on the surface might originate from any position underneath the same surface area around this point. Therefore $l^{*}$ sets the lower bound for the spatial resolution of wide field diffuse reflectance imaging techniques. a)

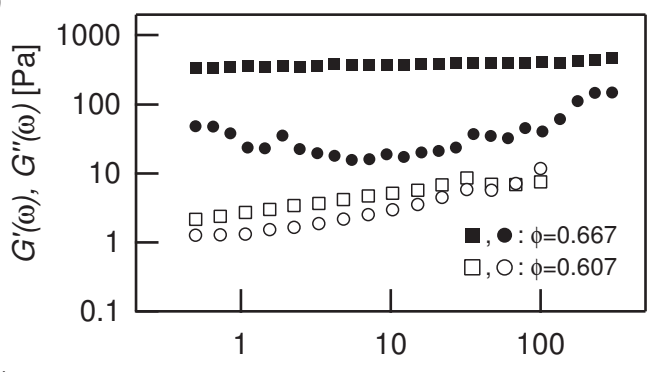

b) $\omega[\mathrm{rad} / \mathrm{s}]$

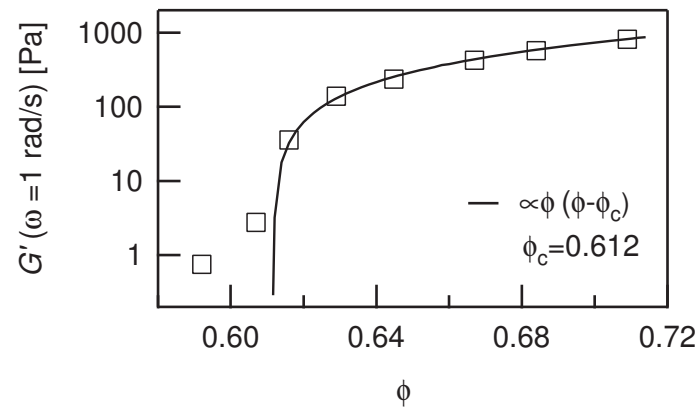

FIGURE 4. a) Results for the elastic (squares) and loss (circles) moduli, $G^{\prime}(\omega)$ and $G^{\prime \prime}(\omega)$, measured for two solid samples. b) Volume fraction dependence of the elastic shear modulus G' measured at $\omega=1 \mathrm{rad} / \mathrm{s}$ for dense emulsions. The solid line is a fit to the network spring model yielding a critical volume fraction of $\phi_{c}=0.612$.

\section{RESULTS AND DISCUSSION}

The origin of elasticity in dense repulsive emulsions depends on whether individual droplets are deformed or not. At volume fractions close to the glass transition of hard-spheres, the elasticity is entropic in origin and arises from the frustration of droplet's configurations due to crowding. Above a critical volume fraction $\phi_{c}$, droplets start to deform and flat facets are created between droplets. Due to the surface tension $\sigma$, elastic energy can be stored at the droplet's interfaces and a shear perturbation to the system increases the potential energy with respect to the un-sheared state. In this regime, the volume fraction dependence of the elastic shear modulus of monodisperse emulsions is well captured by the network spring model predicting $G^{\prime} \sim \phi\left(\phi-\phi_{c}\right)[7,15]$. Here, $\phi_{c}$ is the volume fraction at which the deformation of droplets become the dominant component of the elasticity. The model assumes the existence of a stress bearing network composed of a number of droplet that varies linearly with $\left(\phi-\phi_{c}\right)$, as well as an equivalent deformation for droplets within the the network.

The results for the elastic and loss moduli, $G^{\prime}(\omega)$ and $G^{\prime \prime}(\omega)$, are presented in the Figure 4a) for samples at $\phi=0.607$ and 0.667 . For both samples, the mechani- 


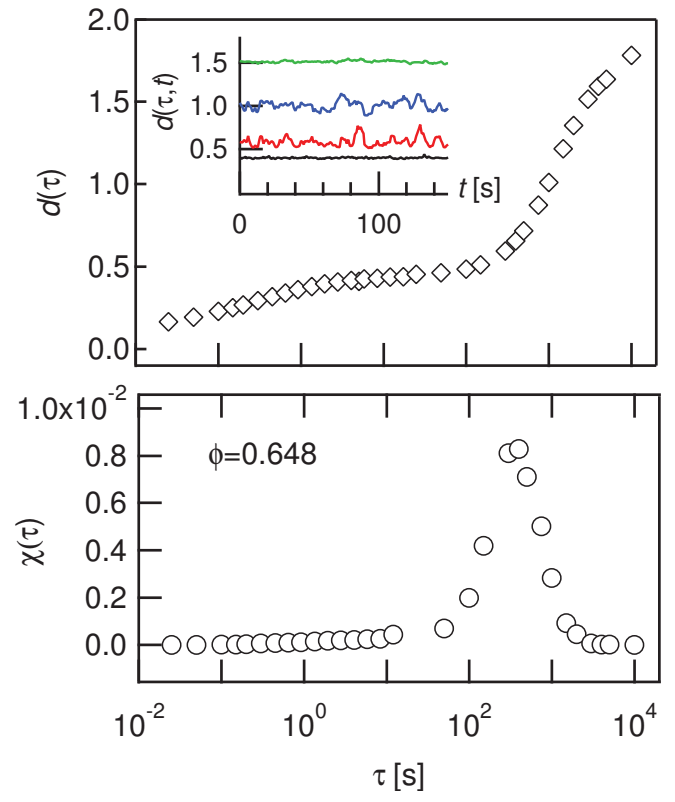

FIGURE 5. a) Structure function $d(\tau)$ measured using near field diffusion wave spectroscopy for an emulsions at $\phi=$ 0.648. Inset: Instantaneous degree of structure $d(\tau, t)$ as a function of time for different lag times $\tau=5,75,300$ and 750s. b) Normalized variance $\chi(\tau)$ of the of the instantaneous degree of structure.

cal response is dominantly elastic at low frequencies. In Figure 4 we report the volume fraction dependence of the elastic modulus $G^{\prime}$ measured at $1 \mathrm{rad} / \mathrm{s}$ for all densities studied. We observe an initial sharp rise of the elastic modulus, followed by a regime where the elastic modulus increases almost linearly with volume fraction. A fit of the data to the network spring model yields a critical volume fraction of $\phi_{c}=0.612$ (see Fig. 4). Four our system, droplets therefore start to experience deformation above a volume fraction of about 0.61 . If one considers an effective diameter that accounts for the short range Coulomb repulsion present between droplets, we can estimate an effective contact volume fraction $\phi_{c}^{\text {eff }} \approx 0.624$. This value is very close to the expected jamming transition of spheres $\phi_{J} \simeq 0.64$.

In the following, we describe general features of the microscopic dynamics observed for dense emulsion having $\phi>\phi_{c}$. The dynamics is measured using diffusing wave spectroscopy with a near field detection scheme [17]. The temporal fluctuation of the dynamics are characterized by computing the spatial average of the structure function $d(\tau, t)=\langle d(\mathbf{r}, \tau, t)\rangle_{\mathbf{r}}$ and its statistical properties (such as the variance of fluctuations). Here $\langle\ldots\rangle_{i}$ indicates the averaging over the variable $i$. In our experiments the dynamics reaches a stationary state after a waiting time of about $t=10$ hours. From this point on we can time-average the measured intensity trace and correlations and we can then reproducibly determine the lag time dependence of the structure function $d(\tau)=\langle d(\mathbf{r}, \tau, t)\rangle_{\mathbf{r}, t .}$. In fig. 5 we present $d(\tau, t)$ and $d(\tau)$ obtained for a sample at $\phi=0.648$. The structure function $d(\tau)$ exhibits a plateau at intermediate lag times $\tau$, extending up to $500 \mathrm{~s}$, and is followed by a long time relaxation towards its maximum value of 2 .

At this volume fraction, the motion of a droplet is limited by direct contact forces with neighboring droplets; the elasticity of the emulsion is dominated by compression of droplets. The presence of dynamical heterogeneities in the relaxation of emulsions can be studied by calculating the normalized variance $\chi(\tau)=$ $\sigma_{t}^{2}(d(\tau, t)) / d^{2}(\tau, t)$. The normalized variance $\chi$ is related to the dynamic susceptibility $\chi_{4}$, introduced in studies of the glass transition in supercooled liquids and colloids $[24,20]$. The results for $\chi(\tau)$ are presented in the Fig $5 \mathrm{~b}$ ) for the volume fraction corresponding to the upper panel. $\chi$ exhibits a marked peak around the terminal relaxation time of the system, supporting the presence of heterogeneous dissipative processes at these times scales.

As shown above the network spring model successfully describe the rheology of compressed emulsions above $\phi_{c}$. The model assumes local gradients in the volume fraction of the droplets in order to form a stress bearing network. Unbalanced forces acting on the network could lead to local relaxations of droplets configurations, hence providing a mechanism for the observed dynamical heterogeneities. Our experiments also permit to study the spatial fluctuations of the dynamics. Indeed, the instantaneous structure function $d(\mathbf{r}, \tau, t)$ measured image plane provides both temporal and spatial resolu-

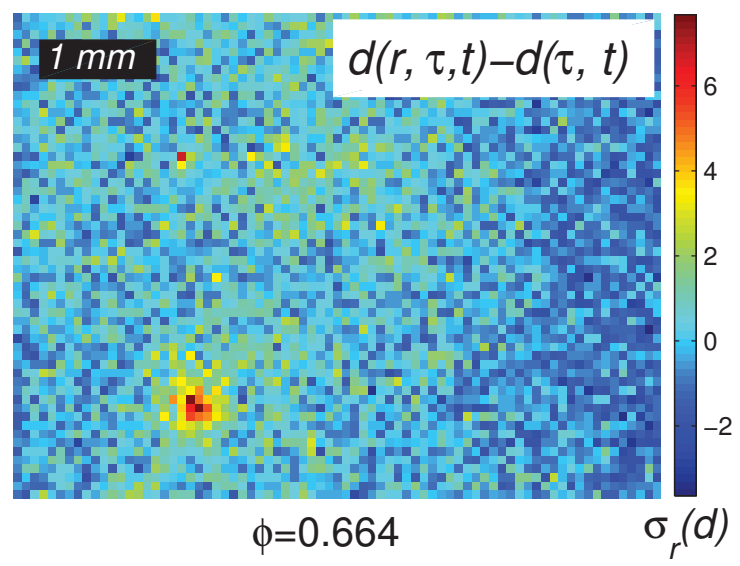

FIGURE 6. Dynamical activity map displaying the deviation of the intensity structure function $d(\mathbf{r}, \tau=5.2 \mathrm{~s}, t)$ from its spatial average value in units of the standard deviation $\sigma_{\mathbf{r}}$ for a sample at $\phi=0.664$. 
tion $[17,19]$. In the following, we fix the lag time $\tau$ to a value well within the plateau of the ensemble averaged structure function. We then measure $d(\mathbf{r}, \tau, t)$ as function of time $t$ in order to obtain a large amount of two- dimensional mapping of the dynamics. An example of an activity map $d(\mathbf{r}, \tau, t)$ for $\tau=5.2 \mathrm{~s}$ is presented in Figure 6. Regions of higher/lower activity are clearly visible (warm/cold color). Our measurements therefore represent a direct confirmation of the heterogeneous nature of the relaxation processes involved in jammed assembly of emulsion droplets. The lateral extent of the observed spatial heterogeneities is of the order of several tens or hundreds of microns, intermediate between the droplet size of $2 \mu \mathrm{m}$ and the macroscopic sample size of about $10 \mathrm{~mm}$.

\section{CONCLUSION}

We have reported on the heterogeneous dynamics in a compressed emulsion using spatially resolved light scattering. The spatial resolution resolution of the experiment is set by the sample turbidity and in our case is of the order of 30-40 $\mu \mathrm{m}$, corresponding to about 20 droplet diameters. Monitoring the dynamic properties on such length scales, previously not accessible to light scattering techniques, will be of prime importance for future scattering studies of the glassy dynamics of dense colloidal systems.

\section{ACKNOWLEDGMENTS}

This project has been financially supported by the Swiss National Science Foundation (Project Nr. 132736 and 123104) and the Adolphe Merkle Foundation.

\section{REFERENCES}

1. Mason, T.G., et al., Monodisperse Emulsions: Properties and uses, in Encyclopedia of Emulsion Technology, B. P., Editor. 1996, Marcel Dekker: New York, Basel, Hong Kong. p. 299.

2. T. G. Mason et al., J. Phys.:Condens. Matter 18,R635-R666 (2006).

3. J. Bibette, D. Roux and F. Nallet, Phy. Rev. Lett. 65, 2470 (1990).

4. J. Bibette, Depletion Interactions and Fractionated Crystallization for Polydisperse Emulsion Purification, Journal of Colloid and Interface Science 147, 1991, 474-478

5. F. Scheffold et al., Phy. Rev. Lett. 104, 128304 (2011).

6. D. A. Sessoms et al.,Phil. Trans. R. Soc. A 367, 5013 (2009).

7. T. G. Mason, J. Bibette, and D. A. Weitz, Phy. Rev. Lett. 75, 2051 (1995).
8. H. Gang et al., Phys. Rev. E 59, 715 (1999).

9. J. Brujic et al., Phy. Rev. Lett. 98, 248001 (2007).

10. I. Jorjadze et al., PNAS 108, 4286 (2011).

11. L. J. Teece, M. A. Faers and P. Bartlett, Soft Matter 7, 1341 (2011).

12. S. S. Datta et al., Phy. Rev. E 84, 041404 (2011).

13. In a more rigereous approach a maximally random jammed (MRJ) state can been introduced to replace the random close packing picture [14].

14. S. Torquato, T. M. Truskett, P.G. Debenedetti, Phys. Rev. Lett. 84, 2064 (2000).

15. M. D. Lacasse et al., Phy. Rev. Lett. 76, 3448 (1996).

16. T. G. Mason et al.,Phys. Rev. E 56, 3150 (1997).

17. P. Zakharov and F. Scheffold, Soft Materials 8, 102 (2010).

18. S. E Skipetrov et al., Opt. Express 18, 14519 (2010).

19. J. Peuser, F. Cardinaux R. Cerbino, P. Zakharov and F. Scheffold, preparation

20. L. Cipelletti and E. R. Weeks, Glassy dynamics and dynamical heterogeneity in colloids. Chapter 4 of Dynamical Heterogenity in glasses, colloids and granular matter, L. Berthier, B. Biroli, J.-P. Bouchaud, L. Cipelletti, W. van Saarloos Eds, Oxford University Press, Owford (2011)

21. K. Schätzel, J. Mod. Opt 30, 155 (1983).

22. M. Erpelding, A. Amon and J. Crassous, Phys. Rev. E 78, 046104 (2008).

23. A. Duri et al., Phys. Rev. Lett. 102, 085702 (2009).

24. D. Lacevic et al., J. Chem. Phys. 119, 7372 (2003). 